\title{
Universiteit
}

Leiden

The Netherlands

\section{Momentum noise in a quantum point contact}

Beenakker, C.W.J.; Tajic, A.; Kindermann, M.

\section{Citation}

Beenakker, C. W. J., Tajic, A., \& Kindermann, M. (2002). Momentum noise in a quantum point contact. Retrieved from https://hdl.handle.net/1887/1274

Version: $\quad$ Not Applicable (or Unknown)

License: $\quad$ Leiden University Non-exclusive license

Downloaded from: https://hdl.handle.net/1887/1274

Note: To cite this publication please use the final published version (if applicable). 


\title{
Momentum noise in a quantum point contact
}

\begin{abstract}
A Tajı, $M$ Kındermann, and C W J Beenakkeı
Instituut Lorentz Universiteit Leiden PO Box 9506, 2300 RA Leiden The Netherlands

(Received 17 June 2002, revised manuscript received 19 September 2002, publıshed 13 December 2002)

Ballıstic elections flowing through a constiction can transfer momentum to the lattice and excite a vibration of a free-standing conductor We show (both numenically and analytically) that the electromechanical noise power $P$ does not vanish on the plateaus of quantized conductance - in contrast to the current norse The dependence of $P$ on the constriction width can be oscillatory or stepwise, depending on the geometry The stepwise increase amounts to an approximate quantization of momentum noise
\end{abstract}

DOI 10 1103/PhysRevB 66 2413XX

PACS number(s) $8585+$, $7323 \mathrm{Ad}, 7350 \mathrm{Td}, 7363 \mathrm{Rt}$

Not long after the discovery of conductance quantization in a ballistic constiıction ${ }^{1}$ it was predicted that the quantization is noiseless ${ }^{2}$ The time dependent current fluctuations should vanish at low temperatures on the plateaus of quantized conductance and they should peak in the tiansition from one plateau to the next The conclusive experimental verification of this piediction followed many years late,${ }^{3}$ delayed by the difficulty of elımınating extraneous sources of noise The notion of noiseless quantum ballistic tiansport is now well established ${ }^{4}$

The origin of noiseless transpoit lies in the fact that the eigenvalues $T_{n}$ of the transmission matıx product $t t^{\dagger}$ take only the values 0 or 1 on a conductance plateau The cuncent norse power at zero temperatue $P_{l} \propto \sum_{n} T_{n}\left(1-T_{n}\right)$ then vanishes ${ }^{5}$ In other woids, current fluctuations require paitrally filled scatten ing channels, which are incompatible with a quantized conductance

In this paper we point out that the notion of noiseless quantum ballistic tianspoit does not apply if one consideis momentum transfer instead of charge tiansfer Momentum norse created by an electiral curient (socalled electiomechanical norse) has been studied in the tunneling regime ${ }^{6}$ and in a diffusive conductor, ${ }^{7}$ but not yet in connection with ballistic transport Our analysis is based on a recent scatteing matrix repiesentation of the momentum norse power $P$, according to which $P$ depends not only on the transmission eigenvalues but also on the eigenvectors ${ }^{8}$ This makes it possible for the elections to generate noise even in the absence of partially filled scattering channels

The geometiy is shown schematically in Fig 1 We consider a two-dimensional election gas channel in the $x-y$ plane The width of the channel in the $y$ diection is $W$ and the length in the $x$ direction is $L$ The channel contains a nairow constiction of length $\delta L \ll L$ and width $\delta W \ll W$ located at a distance $L^{\prime}$ from the left end (We choose $x=0$ at the middle of the constiction, so that the channel extends fiom $-L^{\prime}<x<L-L^{\prime}$ ) A voltage $V$ drives a curient thiough the constriction, exciting a vibiation of the channel We seek the low-frequency norse power

$$
P=2 \int_{-\curvearrowright}^{\curvearrowright} d t \overline{\delta F(0) \delta F(t)}=\lim _{t \rightarrow \infty} \frac{2}{t} \overline{\Delta \mathcal{P}(t)^{2}}
$$

of the fluctuating force $\delta F(t) \equiv F(t)-\bar{F}$ that dives the v1biation The noise power is propottional to the variance of the momentum $\Delta \mathcal{P}(t)$ transferied by the electrons to the channel in a long time $t$

We assume that the election gas is deposited on top of a doubly clamped beam extended along the $x$ axis and free to vibiate in the $y$ disection The solution of the wave equation is $\mathbf{u}(\mathbf{r}, t)=\hat{y} u(x) \cos \omega t$, with $\omega$ the mode fiequency and $u(x)$ the mode profile Both $u$ and $d u / d x$ vanish at the ends of the beam and $u(x)$ is noimalized such that it equals to 1 at the point $x_{0}$ at which the amplitude is measured ${ }^{7}$ We choose $x_{0}=0$ so that $F$ corresponds to a point force at the location of the constiction

The wave functions are 1epresented by scatterng states The incident wave has the form $\phi_{n}(\mathbf{r})$ $=\left|\hbar k_{n} / m^{4}\right|^{-1 / 2} \exp \left(i k_{n} \lambda\right) \Phi_{n}(y)$, where $m^{+}$is the effective mass, $n=1,2, \quad, N$ is the mode index, $\Phi_{n}$ the transverse wave function, and $k_{n}= \pm\left(2 m^{*} / \hbar^{2}\right)^{1 / 2}\left(E_{F}-E_{n}\right)^{1 / 2}$ the longitudinal wave vector (at Feimi energy $E_{\Gamma}$ laiger than the cutoff energy $E_{n}$ ) We take $k_{n}$ positive to the left and negative to the 11ght of the constiction Incident and outgoing waves are related by the $2 N \times 2 N$ unitaly scattening mat1 $1 \mathrm{x}$

$$
S=\left(\begin{array}{ll}
r & t^{\prime} \\
t & t^{\prime}
\end{array}\right),
$$

containing the $N \times N$ transmission and reflection matrices $t, t^{\prime}, 1, r^{\prime}$ We assume time reversal symmetry, so that $\Phi_{n}$ is real and $S$ is symmetric

As delived in Ref 8 , the norse power $P$ and the mean force $\bar{F}$ for a localized scatterer can be expressed in terms of the matix $S$ and a Hermitian matrix $\mathcal{M}_{n n^{\prime}}$

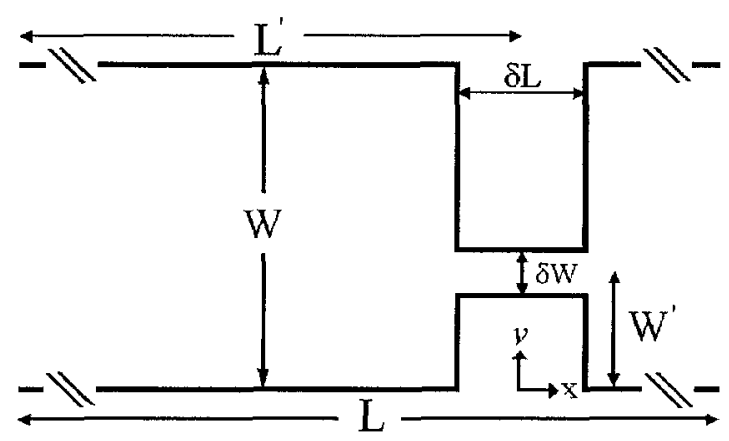

FIG 1 Schematic diagiam of a two-dimensional channel con taıning a constutction 
$=m^{*-1} \Sigma_{\alpha \beta}\left\langle n\left|p_{\alpha} u_{\alpha \beta} p_{\beta}\right| n^{\prime}\right\rangle$ of expectation values in the basis of incident modes. The expectation value is taken of the electron momentum flux $m^{*-1} p_{\alpha} p_{\beta}$, weighted by the strain tensor $u_{\alpha \beta}=\frac{1}{2}\left(\partial u_{\alpha} / \partial x_{\beta}+\partial u_{\beta} / \partial x_{\alpha}\right)$. The matrix $\mathcal{M}$ is block diagonal,

$$
\mathcal{M}=\left(\begin{array}{cc}
M_{\mathrm{L}} & 0 \\
0 & M_{\mathrm{R}}
\end{array}\right) \text {. }
$$

At zero temperature and to first order in the voltage one has, for a twofold spin degeneracy,

$$
\begin{gathered}
P=\frac{4 e V}{h} \operatorname{Tr}\left(r r^{\dagger} M_{\mathrm{L}}^{*} t^{\prime} t^{\prime \dagger} M_{\mathrm{L}}^{*}+r^{\prime} r^{\prime \dagger} M_{\mathrm{R}}^{*} t t^{\dagger} M_{\mathrm{R}}^{*} s\right. \\
\left.-2 t r^{\top} M_{\mathrm{L}}^{*} r t^{\dagger} M_{\mathrm{R}}^{*}\right), \\
\bar{F}=\frac{2 e V}{h} \operatorname{Tr}\left(M_{\mathrm{L}}+r r^{\dagger} M_{\mathrm{L}}^{*}+t t^{\dagger} M_{\mathrm{R}}^{*}\right) .
\end{gathered}
$$

In Eq. (5) we have not included the equilibrium contribution to the mean force (at $V=0$ ). Electron-electron interactions (screening) are not accounted for, since we do not expect any appreciable charge accumulation in a ballistic system.

For a transverse vibration the blocks $M_{\mathrm{L}}, M_{\mathrm{R}}$ have elements

$$
\begin{aligned}
\left(M_{\mathrm{L}, \mathrm{R}}\right)_{n m}= & \frac{\hbar\left(k_{n}+k_{m}\right)}{2 i\left|k_{n} k_{m}\right|} \int d y \Phi_{n}(y) \Phi_{m}^{\prime}(y) \int_{\mathrm{L}, \mathrm{R}} d x u^{\prime}(x) \\
& \times \exp \left[i x\left(k_{m}-k_{n}\right)\right]
\end{aligned}
$$

The integral over $x$ extends over the region $\left(-L^{\prime},-\delta L / 2\right)$ to the left of the constriction for $M_{\mathrm{L}}$ and over the region $\left(\delta L / 2, L-L^{\prime}\right)$ to the right of the constriction for $M_{\mathrm{R}}$. We abbreviate $q_{n m}=k_{m}-k_{n}$. For $|n-m|$ of order unity one has $q_{n m}$ of order $1 / W$, so that the range of $x$ that contributes to the integral is of order $W$. [Contributions from inside the constriction are smaller by a factor $\min (\delta W, \delta L) / W$.] Since $W$ is much greater than the Fermi wave length $\lambda_{F}$, we are justified in using the asymptotic plane wave form of the scattering states to calculate $\mathcal{M}$.

We take hard-wall boundary conditions at $y=0, W$, hence $\Phi_{n}(y)=(2 / W)^{1 / 2} \sin (n \pi y / W), \quad E_{n}=\left(\hbar^{2} / 2 m^{*}\right)(n \pi / W)^{2}, \quad N$ $=\left[2 W / \lambda_{F}\right]$, and $\left(k_{n}+k_{m}\right) q_{n m}=(\pi / W)^{2}\left(n^{2}-m^{2}\right)$. The overlap $\int_{0}^{W} d y \Phi_{n} \Phi_{m}^{\prime}$ is evaluated straightforwardly, but the integration over $x$ requires more care. The derivative $u^{\prime}(x)$ $=d u / d x$ of the mode profile vanishes at the two clamped ends of the beam, as well as at its center. We assume that the constriction is off-center, therefore $u^{\prime}( \pm \delta L / 2) \approx u^{\prime}(0) \neq 0$. We write $u^{\prime}(0)=u_{0} / L$, with $u_{0}$ a number of order unity. Upon partial integration we find, to first order in $W / L$,

$$
\begin{aligned}
\int_{L, \mathrm{R}} d x u^{\prime}(x) \exp \left(l x q_{n m}\right)= & \pm \frac{u_{0}}{i q_{n m} L} \exp \left(\mp l q_{n m} \delta L / 2\right) \\
& +\mathcal{O}(W / L)^{2}
\end{aligned}
$$
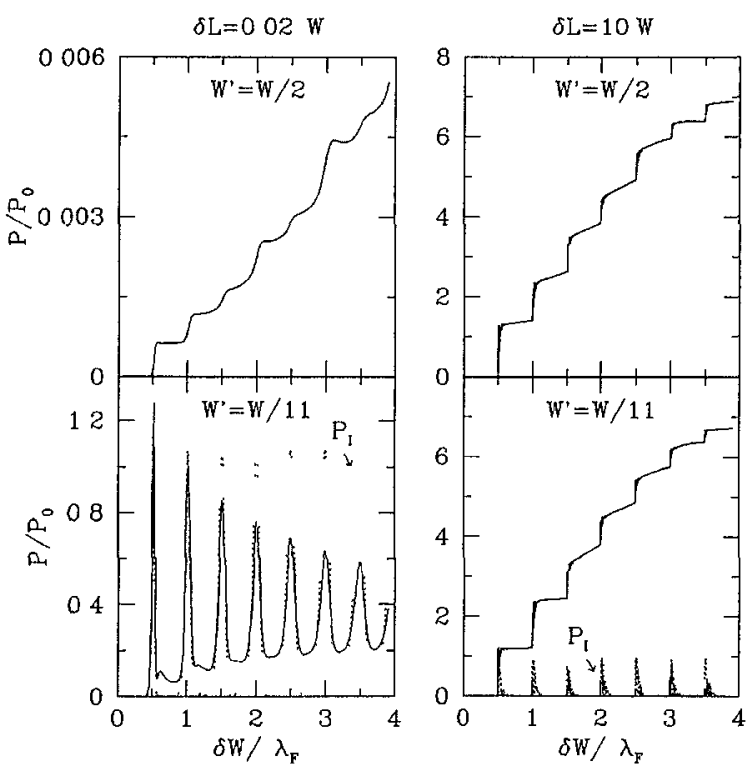

FIG. 2 Solid lines: noise power $P$ for transverse vibration versus width of constriction $\delta W$, at fixed width $W=49.9 \lambda_{F}$ of the wide channel. The left panels are for a short constriction with and without axial symmetry. The right panels are the corresponding results for a long constriction. The dotted line is the current noise $P_{I}$ in units of $e^{3} V / h$ (which is nearly the same with and without axial symmetry).

(The upper sign is for region $\mathrm{L}$, the lower sign for region R.) We thus arrive at $M_{\mathrm{L}}=-M_{\mathrm{R}} \equiv M$, with

$$
\begin{aligned}
M_{n m}= & \frac{2 \hbar W u_{0}}{\pi^{2} L}\left(\sigma_{n m}-1\right) \frac{n m\left(k_{n}+k_{m}\right)^{2}}{\left(n^{2}-m^{2}\right)^{2}\left|k_{n} k_{m}\right|^{1 / 2}} \\
& \times \exp \left[i\left(\left|k_{n}\right|-\left|k_{m}\right|\right) \delta L / 2\right] .
\end{aligned}
$$

The symbol $\sigma_{n m}=\frac{1}{2}\left[1+(-1)^{n+m}\right]$ selects indices of the same parity, so that $M_{n m}=0$ if $n$ and $m$ are both even or both odd.

Our constriction has left-right symmetry, so $r=r^{\prime}$ and $t$ $=t^{\prime}$. We contrast the case $W^{\prime}=\frac{1}{2} W$ of axial symmetry with the case $W^{\prime} \ll \frac{1}{2} W$ of a constriction placed highly off-axis. We also contrast the short-constriction case $\delta L \ll W$ (point contact geometry) with the long-constriction case $\delta L \gg W$ (microbridge geometry). The reflection and transmission matrices are calculated by matching wave-function modes at $x$ $= \pm \delta L / 2$, cf. Ref. 9 .

In Fig. 2 we show the dependence of the transverse noise power $P$ [in units of $P_{0}=(4 e V / h)\left(N u_{0} \hbar / L\right)^{2}$ ] on the width $\delta W$ of the constriction, at fixed width $W$ of the wide channel. (We choose $W=49.9 \lambda_{F}$, so $N=99$.) The average transverse force $\bar{F}$ is shown in Fig. 3 , normalized by $F_{0}$ $=(2 e V / h)\left(N u_{0} \hbar / L\right)$. (Note that $\bar{F}=0$ for the axially symmetric case.) The conductance $G=\left(2 e^{2} / h\right)$ Tr $t t^{4}$ and the current noise $P_{I}=\left(4 e^{3} V / h\right) \operatorname{Tr} t t^{\prime}\left(1-t t^{\dagger}\right)$ are included in these plots for comparison.

The plots show a remarkably varied behavior: For the short constriction without axial symmetry the norse power $P$ of the transverse force oscillates as a function of the constric- 

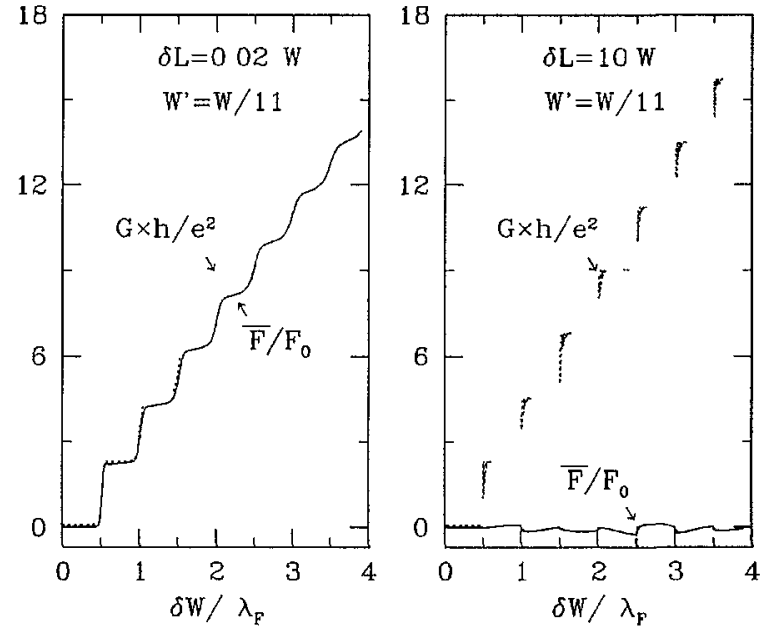

FIG 3 Solid lines average transverse force as a function of constriction width, in the absence of axial symmetry (positive values point in the positive $y$ direction in the geometry of $F_{1} g$, for a current flowing in the positive $x$ direction) The left and right panels are for a short and long constriction, respectively The conductance of the constriction is shown as a dotted line. The average transverse force is identically zero for the axially symmetric geometry ( $W^{\prime}$ $=W / 2)$

tion width $\delta W$, in much the same way as the current noise power $P_{I}$ oscillates. ${ }^{2,5}$ However, the minima in $P$ do not go to zero like the minima in $P_{I}$, demonstrating nonzero momenturn noise on the plateaus of quantized conductance. If the short constriction is precisely at the center of the channel, $P$ increases nearly monotonically with $\delta W$. For a long constriction $P$ increases nearly monotonically regardless of whether there is axial symmetry or not. The increase of the noise power is stepwise, reminiscent of the conductance. (The current noise in the long constriction remanns osc1llatory.) The mean transverse force behaves similarly to the conductance for the short constriction, but fluctuates around zero for the long constriction.

In order to explain the approximate quantization of momentum norse in analytical terms it is convenient to decompose the (symmetric) transmission matrix as $t_{n m}$ $=\Sigma_{n^{\prime}} U_{n n^{\prime}} U_{m n^{\prime}} \sqrt{T_{n^{\prime}}}$, where $U$ is an $N \times N$ unitary matrix and $T_{n} \in[0,1]$ is the transmission eigenvalue (eigenvalue of $t t^{\mathrm{T}}$ ). Similarly, the reflection matrix is decomposed as $r_{n m}$ $=i \Sigma_{n^{\prime}} U_{n n^{\prime}} U_{m n^{\prime}} \sqrt{1-T_{n^{\prime}}}$. In this representation Eq. (4) takes the form

$$
\begin{aligned}
P= & \frac{8 e V}{h} \sum_{n, m}\left|X_{n m}\right|^{2}\left\{\left(1-T_{n}\right) T_{m}+\left[T_{n}\left(1-T_{n}\right) T_{m}\right.\right. \\
& \left.\left.\times\left(1-T_{m}\right)\right]^{1 / 2}\right\}, \quad X=U^{\dagger} M^{*} U .
\end{aligned}
$$

The matrix $M$ couples only mode indices of different parity, cf. Eq. (8). The presence or absence of axial symmetry manifests itself in the matrix $U$, which couples only indices of the same parity if $W^{\prime}=W / 2$. In this axially symmetric case $X_{n m}=0$ if $n, m$ have the same parity.

In a simple model ${ }^{10}$ of a long and narrow ballistic microbridge, $U$ is a random matrix while the tiansmission eigen- values take on only two values: $T_{n}=1$ for $1 \leqslant n \leqslant \delta N$ and $T_{n}=0$ for $\delta N<n \leqslant N$. The number $\delta N=\left[2 \delta W / \lambda_{F}\right]$ is the quantized conductance of the constriction (in units of $\left.2 e^{2} / h\right)$. Averages of $U$ over the unitary group introduce Kronecker delta's (cf. App. B in Ref. 10). We need the average $\Omega_{p p^{\prime} q q^{\prime} n m} \equiv\left\langle U_{p n}^{*} U_{q m}^{*} U_{p^{\prime} m} U_{q^{\prime} n}\right\rangle$, given by

$$
\Omega_{p p^{\prime} q q^{\prime} n m}=\frac{1}{N^{2}-1}\left(\delta_{p q^{\prime}} \delta_{q p^{\prime}}-\frac{1}{N} \delta_{p p^{\prime}} \delta_{q q^{\prime}}\right) \text { if } n \neq m,
$$

in the case of broken axial symmetry and

$$
\Omega_{p p^{\prime} q q^{\prime} n m}=\frac{4}{N^{2}-\sigma_{N 1}} \delta_{p q^{\prime}} \delta_{q p^{\prime}} \sigma_{p n} \sigma_{q m} \text { if } \quad \sigma_{n m}=0
$$

in the axially symmetric case.

Substituting these values of $T_{n}$ into Eq. (9) and averaging over $U$ with the help of Eqs. (10) and (11), we find

$$
\begin{aligned}
P & =\frac{8 e V}{h} \sum_{n=\delta N+1}^{N} \sum_{m=1}^{\delta N} \sum_{p, p^{\prime}, q, q^{\prime}=1}^{N} \Omega_{p p^{\prime} q q^{\prime} n m^{\prime}} M_{p p^{\prime}}^{*} M_{q^{\prime} q} \\
& =\frac{8 e V}{h} \frac{\delta N}{N}\left(1-\frac{\delta N}{N}\right) \operatorname{Tr} M^{2}=\frac{\pi^{2}}{9} \delta N P_{0}, \quad N \gg \delta N,
\end{aligned}
$$

regardless of whether axial symmetry is present or not. We thus obtain a stepwise increase of $P$ as a function of $\delta W$ with step height $\Delta P=\left(\pi^{2} / 9\right) P_{0}$. The numerically obtaned step height in Fig. 2 agrees within $10 \%$ with the analytical estimate for the first step. For subsequent steps the agreement becomes worse, presumably because the approximation of a uniform distribution of $U$ breaks down as $\delta W$ increases. We can also calculate the mean transverse force in the same way,
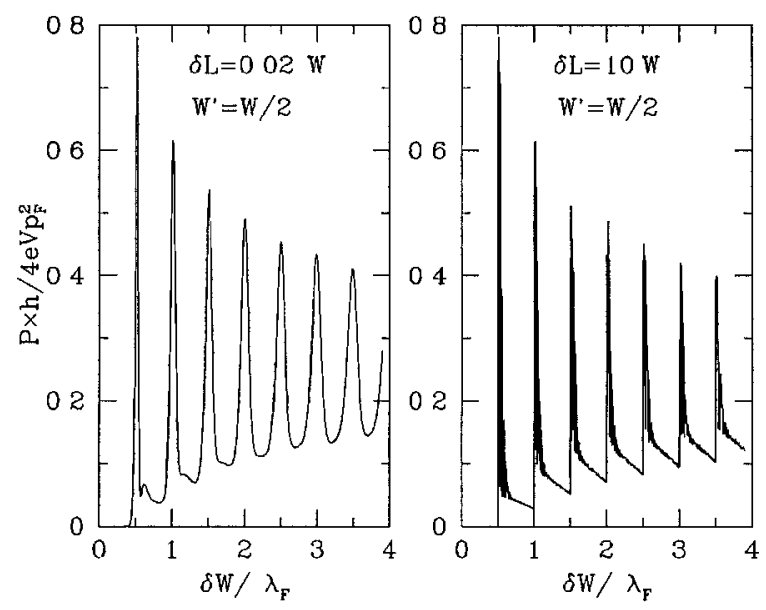

FIG 4. Noise power for longitudinal vibration of a short constriction (left panel) and a long constriction (right panel) These plots are for $W^{\prime}=W / 2$, but thete is no noticeable dependence on the 1atio $W^{\prime \prime} / W$ The mean longitudinal force (not shown) decreases stepwise as a function of $\delta W$ in both the short and long constriction. 
starting fiom Eq (5), and find $\bar{F} \propto \operatorname{Tr} M=0$, in accordance with the numenical result that $\bar{F} \ll F_{0}$ for a long constiction

In the short-constiuction case $\delta L \ll W$ we may not tieat $U$ as uniformly distributed in the unitary group, and this has prevented us from finding a simple analytical iepresentation of the numerical data

This rich geometry dependence of the noise powet is characteristic for a transverse vibiation For comparison we discuss the case of a longitudinal vibiation, corresponding to a mode profile $\hat{x} u(x)$ oriented along the direction of the cur1ent through the constriction (instead of perpendicular to it) Such a longitudinal vibration coriesponds to a compression mode of the beam, which is at a higher fiequency than the bending mode excited by a transverse vibration For a long1tudinal vibration the matrices $M_{\mathrm{L}}, M_{\mathrm{R}}$ are diagonal $\left(M_{\mathrm{L}}\right)_{n m}=-\left(M_{\mathrm{R}}\right)_{n m}=\delta_{n m} \hbar\left|k_{n}\right| u(0)$ We take $u(0)=1$

The norse power is plotted in $\mathrm{F}_{1} \mathrm{~g} 4$ for both a long and a short constriction It does not depend on the presence or absence of axial symmetiy and is also rathei insensitive to the length of the constiction The order of magnitude of the longitudinal noise power is $(4 e V / h) p_{F}^{2}$, with $p_{F}=\hbar k_{F}$ the Fermi momentum This is laiger than the typical transveise nowse power $P_{0}$ by a factor of order $\left(k_{F} L / N\right)^{2} \simeq(L / W)^{2}$ Inserting parameters $V=1 \mathrm{mV}, k_{F}=10^{8} \mathrm{~m}^{-1}$, typical for a two-dimensional electron gas, one estimates $(4 e V / h) p_{F}^{2}$ $\simeq 10^{-40} \mathrm{~N}^{2} / \mathrm{Hz}$ This is below the force sensitivity of piesent day nanomechanical oscillators, but is hoped to be ieached in future generations of these devices ${ }^{11}$

In summary, we have demonstrated that the momentum noise of ballistic electrons does not vanish on the plateaus of quantized conductance Conductance quantization iequires absence of backscattering in the constiction, but it does not preclude inter-mode scattering Momentum norse makes this inte1-mode scattering visible in a way that current noise can not The dependence of the momentum norse on the constiction width was found to be remarkably varied, ranging fiom oscillatory to stepwise, depending on the direction of the vibration (longitudinal or transverse to the constiction), the presence or absence of axial symmetiy, and the length of the constriction The stepwise increase amounts to a quantum of momentum noise that might be observable with an ultiasensitive oscillator

Th1s 1esea1ch was supported by the Dutch Science Foundation NWO/FOM
${ }^{1} \mathrm{~B} J$ van Wees, $H$ van Houten, C W J Beenakker, J G Willıamson, L P Kouwenhoven, D van der Marel, and C T Foxton, Phys Rev Lett 60, 848 (1988), D A Wharam, T J Thornton, R Newbury, M Pepper, H Ahmed, J E F Frost, D G Hasko, D C Peacock, D A Ritchie, and G A C Jones, J Phys C 21, L209 (1988)

${ }^{2}$ G B Lesovik, Pis'ma Zh Eksp Teor Fiz 49, 513 (1989) [JETP Lett 49, 592 (1989)]

${ }^{3}$ M I Reznikov, M Heiblum, H Shtrikman, and D Mahalu, Phys Rev Lett 75, 3340 (1995), A Kumar, L Samınadayar, D C Glattli, Y Jın, and B Etienne, lbıd 76, 2778 (1996)

${ }^{4}$ Ya M Blanter and M Buttıker, Phys Rep 336, 1 (2000)
${ }^{5} \mathrm{M}$ Buttıker, Phys Rev Lett 65, 2901 (1990)

${ }^{6}$ M F Bocko, KA Stephenson, and R H Koch, Phys Rev Lett 61, 726 (1988), B Yurke and G P Kochansk1, Phys Rev B 41, 8184 (1990)

${ }^{7}$ A V Shytov, L S Levitov, and C W J Beenakker, Phys Rev Lett 88, 228303 (2002)

${ }^{8} \mathrm{M}$ Kundermann and C W J Beenakke1, cond-mat/0206403, Phys Rev B (to be published)

${ }^{9}$ A Szafer and A D Stone, Phys Rev Lett 62, 300 (1989)

${ }^{10}$ C W J Beenakker, Rev Mod Phys 69, 731 (1997)

${ }^{11} \mathrm{M}$ Roukes (private communication) 